$(\mathrm{OR}=1.89,95 \%$ CI $1.47 ; 2.41)$. Prospectively, perceived sex discrimination was associated with increased psychological distress $(B=0.66,95 \%$ CI $0.07 ; 1.24)$, poorer mental functioning $(B=-1.37,95 \%$ CI $-2.71 ;-0.03)$ and lower life satisfaction $(B=-0.32$, 95\% CI $-0.58 ;-0.05)$ over four-year follow-up.

Conclusion Women in the UK who perceive that they have been discriminated against on the basis of their sex report poorer mental health and wellbeing than those who do not perceive sex discrimination. These results provide cross-sectional and prospective evidence of associations between perceived sex discrimination and mental health and wellbeing outcomes in UK adults.

\section{RF09 SOCIO-ECONOMIC INEQUALITIES IN SUICIDE: CAUSATION OR CONFOUNDING? A MORTALITY FOLLOW-UP OF FORTY POPULATION CENSUSES FROM TWELVE EUROPEAN COUNTRIES}

V Lorant*. Institute of Health and Society, UCLouvain, Brussels, Belgium

10.1136/jech-2019-SSMabstracts.124

Background Socio-economic inequalities in suicide remain substantial and persistent in most European countries. The mechanism driving these inequalities, however, remains obscure. Two causal mechanisms have been attributed varying degrees of importance: low socio-economic status may directly increase the risk of suicide (hereafter 'causation') or low socio-economic status and suicide may share confounders (hereafter, 'confounding'). This paper aims to assess whether educational inequalities in suicide (EIS) are due to causation or to confounding.

Methods The DEMETRIQ study collected and harmonised register-based data on mortality follow-up of forty population censuses from fifteen Northern, Southern, Western, and Eastern European populations. More than 89,554 suicides were registered over 300 million person-years. Four tests of causation $v s$ confounding were implemented. Test 1: whether a decreasing (increasing) EIS over the lifecycle supports confounding (or causation). Test 2: whether greater (lower) EIS in males than in females supports confounding (or causation). Test 3: At the country level, whether EIS is more related to the Gini income inequality index (causation) or to the proportion of suicides that take place among the younger age group (confounding). Test 4 applied an instrumental variable approach that exploits changes in the legislation on compulsory educational age to instrument educational status. Tests 1 and 2 were performed with multilevel Poisson Regressions (SAS), test 3 with linear regression, and test 4 used two-stage Poisson Regression (STATA).

Results For test 1, we found that educational inequalities in suicide decreased over the life cycle. The risk of suicide among the less educated as compared to the more highly educated declined from $\mathrm{RR}=1.85$ (95\% CI: 1.6-2.07) in those aged $35-39$ to $\mathrm{RR}=1.27(95 \mathrm{CI} \%, 1.12-1.44)$ for those aged $75-79$. Test 2 indicated that educational inequalities in suicide were systematic and of greater magnitude in males (all countries, $\mathrm{RR}=2.51,95 \% \mathrm{CI}: 2.44-2.58$ ) than in females (all countries $\mathrm{RR}=1.32$, 95\%CI: 1.26-1.38). For test 3, EIS decreased with mean age of suicide (std Beta $=-0.59$, t-test $=-5.0$ ) and increased with the Gini coefficient (std beta $=0.47$, t-test $=2.8$ ). Test 4 indicated that there was no association between higher education and suicide.
Conclusion We found stronger support for the confounding explanation than for causation. Educational inequalities in suicide should be addressed by early targeting of vulnerable groups who struggle to complete their education.

\section{RF10 HIDDEN HARMS OF HYPNOTICS: A POPULATION BASED RECORD LINKAGE STUDY OF PSYCHOTROPIC MEDICATION AND SUICIDE RISK}

${ }^{1}$ IN Onyeka*, 'A Maguire, ${ }^{1,2} \mathrm{D}$ O'Reilly. ${ }^{1}$ Centre for Public Health, Queen's University Belfast, Belfast, UK; ${ }^{2}$ Administrative Data Research Centre Northern Ireland, Centre for Public Health, Queen's University Belfast, Belfast, UK

\subsection{6/jech-2019-SSMabstracts. 125}

Background Suicide is a leading cause of death worldwide with rates of suicide higher in Northern Ireland (NI) compared to the rest of the UK and Ireland. In addition, the consumption of psychotropic medication is increasing. The association between psychotropic medication and suicide risk is contested. Existing population-based studies that have examined whether types of psychotropic medications are associated with suicide are either restricted to older populations or have investigated each medication separately rather than comparing them directly to one another. This study examined the association between psychotropic medication types and death by suicide across the whole adult population.

Methods A cohort of 1,115,647 individuals aged 18-75 was identified from the 2011 Census records, linked to prescription records for the five months surrounding the census and followed until the end of 2015 to determine suicide risk. All individual, household and area-based attributes were derived from census records and selected on the basis of known suicide risk. Five categories of psychotropic medication were identified; none, antidepressants, anxiolytics, hypnotics and antipsychotics. Logistic regression was used to construct models to test the association between specific types of psychotropic medication (singularly and in combinations) and death by suicide adjusting for sociodemographic and physical health characteristics. Several sensitivity analyses were conducted taking into consideration the different timing, amount/threshold of medication and changes in medications consumed.

Results About $14 \%$ of the cohort $(n=153,319)$ had received $\geq 2$ prescriptions of any psychotropic medication in the five months around Census. Eight hundred and seventy persons ( $n=870)$ died by suicide during 2011 to 2015. Suicide was higher in those on psychotropic medication than in those without $(0.24 \%$ versus $0.05 \%$ respectively). In the fully adjusted logistic regression model, association between death by suicide and any psychotropic medication record remained statistically significant $(\mathrm{AOR}=3.69$, 95\%CI: 3.13-4.35). However, individuals solely on hypnotics had the highest AOR of those on singular medication $(\mathrm{AOR}=4.24,95 \% \mathrm{CI}$ : 2.96-6.06 compared to just $\mathrm{AOR}=2.22,95 \% \mathrm{CI}$ : $1.75-2.82$ for those on antidepressant medication only). In terms of medication combinations, use of both anxiolytics and hypnotics $(\mathrm{AOR}=8.76$, 95\%CI: 5.93-12.94) and a combination of antidepressants, anxiolytics and hypnotics $(\mathrm{AOR}=8.53,95 \% \mathrm{CI}$ : 5.62-12.94) were the strongest predictors of deaths by suicide.

Conclusion Risk of death by suicide varied by medication type with hypnotic medications making an important contribution to death by suicide. Although previous work has focused on antidepressant therapy and suicide risk, those on hypnotic medication are an at-risk group that warrant further attention. 